\title{
Uso de Mnemônico como ferramenta de Gestão de Serviços de Saúde para Segurança do Paciente
}

\author{
Use of Mnemonics as a Health Services Management tool for Patient Safety \\ Uso de Mnemonics como herramienta de Gestión de Servicios de Salud para la Seguridad del
}

Paciente

Recebido: 03/07/2021 | Revisado: 10/07/2021 | Aceito: 15/07/2021 | Publicado: 24/07/2021

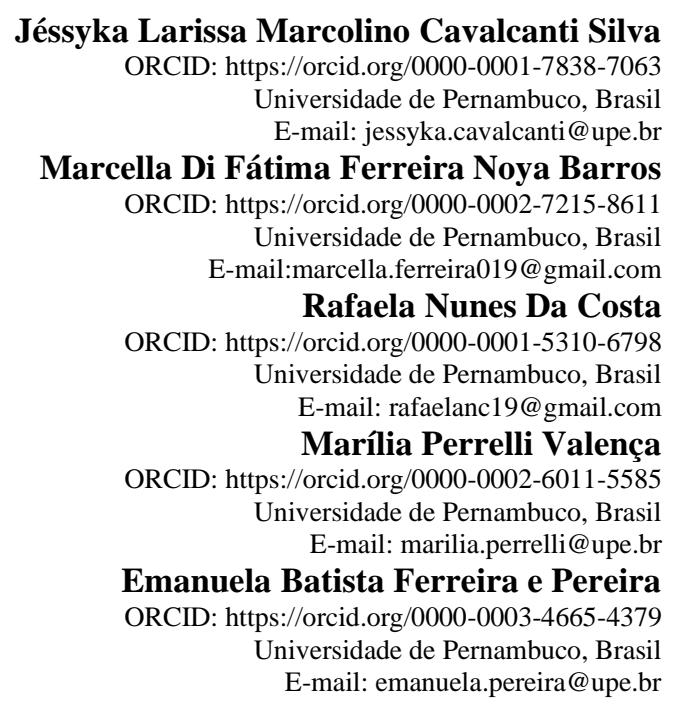

\begin{abstract}
Resumo
Objetivo: Relatar a experiência de acadêmicos de Enfermagem no desenvolvimento de um Mnemônico sobre identificação correta do paciente durante o processo de ensino aprendizagem na unidade temática Gerenciamento em Serviços de Saúde sobre Ferramentas da Gestão da Segurança do Paciente. Método: Trata-se de um relato de experiência, desenvolvido por acadêmicos de uma Universidade pública de Pernambuco, durante o período do ensino remoto em 2020. Resultados: Foi criado um mnemônico, como produto final da atividade com o objetivo de auxiliar os profissionais de saúde na memorização das etapas da assistência ao paciente cirúrgico submetido à transfusão sanguínea. Considerações finais: O mnemônico foi elaborado a fim de assegurar a segurança do paciente, com ênfase na meta 1, submetido a uma transfusão sanguínea no processo cirúrgico representa uma ferramenta prática e acessível no dia a dia das atividades de enfermagem, para otimizar o tempo de atendimento e esquematizar as técnicas de assistência à saúde que favorecem uma maior segurança ao paciente e a equipe.
\end{abstract}

Palavras-chave: Segurança do paciente; Enfermagem; Materiais didáticos; Ensino.

\begin{abstract}
Aims: To report the experience of nursing students in developing a mnemonic about correct patient identification during the teaching-learning process in thematic unit of Management in Health Services on Patient Safety Management Tools. Method: This is an experience report, developed by academics from a public University of Pernambuco, during the period of remote education in 2020. Results: A mnemonic was created, as a final product of an activity, with the aim of helping health professionals in memorizing the steps of safety care for surgical patients undergoing blood transfusion. Final considerations: The mnemonic was designed to ensure patient safety, with emphasis on goal 1, who undergo a blood transfusion in the surgical process represents a practical and accessible tool in the daily routine of nursing activities, to optimize the time of care and outline the health care techniques that improves greater safety for the patient and the team.
\end{abstract}

Keywords: Patient safety; Nursing; Teaching materials; Teaching.

\section{Resumen}

Objetivo: Reportar la experiencia de estudiantes de enfermería en el desarrollo de una mnemotécnica sobre la correcta identificación del paciente durante el proceso de enseñanza-aprendizaje en la unidad temática Gestión en Servicios de 
Salud sobre Herramientas de Gestión de Seguridad del Paciente. Método: Este es un informe de experiencia, desarrollado por académicos de una Universidad pública de Pernambuco, durante el período de educación a distancia en 2020. Resultados: Se creó un mnemónico, como producto final de la actividad, con el objetivo de ayudar a los profesionales de la salud. en la memorización de los pasos del cuidado de los pacientes quirúrgicos sometidos a transfusiones de sangre. Consideraciones finales: El mnemónico fue diseñado para garantizar la seguridad del paciente, con énfasis en la meta 1, quien se somete a una transfusión de sangre en el proceso quirúrgico representa una herramienta práctica y accesible en la rutina diaria de las actividades de enfermería, para optimizar el tiempo de atención y delinear el técnicas asistenciales que favorezcan una mayor seguridad para el paciente y el equipo.

Palabras clave: Seguridad del paciente; Enfermería; Materiales de enseñanza; Enseñando.

\section{Introdução}

Segundo a Classificação Internacional de Segurança do Paciente da Organização Mundial da Saúde em 2014, entendese por Segurança do Paciente a redução a um mínimo aceitável, o risco de dano desnecessário associado ao cuidado de saúde (Ministério da Saúde, 2014). O Programa Nacional de Segurança do Paciente tem como objetivo minimizar possíveis eventos adversos oriundos da atividade laboral dos profissionais de saúde. A incidência de eventos adversos ocasiona o aumento na morbimortalidade, o aumento da permanência da internação dos pacientes e consequentemente nas despesas assistenciais. A assistência segura é uma condição essencial para qualquer terapêutica ou cuidado prestado ao paciente que não pode ser negligenciada (Zampollo, et al., 2018).

O relatório To Err is Human, publicado pelo Institute of Medicine (IOM) dos Estados Unidos da América (EUA), aponta que, aproximadamente, 100.000 pessoas morrem a cada ano em hospitais norte-americanos, decorrentes de eventos adversos preveníveis. A ocorrência desses eventos adversos está relacionada às práticas inadequadas e negligentes de profissionais de saúde e afetam entre 3 e $16 \%$ dos pacientes hospitalizados em países desenvolvidos (Gomes, et al., 2020).

A partir disso, a Organização Mundial da Saúde (OMS) recomenda que as instituições de saúde desenvolvam programas que evidenciem a responsabilidade dos profissionais em relação à segurança do paciente (Zampollo, et al.,2018). Foram estabelecidas pela OMS e preconizadas pela Joint Commisssion International (JCI), 07 (sete) metas internacionais de segurança do paciente, são elas: Identificação correta do paciente; Melhorar a comunicação entre profissionais de saúde; Melhorar a segurança na prescrição, no uso e na administração de medicamentos; Assegurar a cirurgia em local de intervenção, procedimento e paciente corretos; Higienização das mãos para prevenção de infecções relacionadas à assistência à saúde; Reduzir o risco de quedas e úlceras por pressão e Melhorar a segurança na prescrição das solicitações de transfusões de sangue (STS), no uso e na administração de hemocomponentes (Ibsp, 2020).

A primeira meta, Identificação Correta do Paciente, é um processo necessário que assegura ao paciente que a ele seja destinado a um determinado tipo de procedimento ou tratamento. Desde a admissão até a alta hospitalar, os erros de identificação do paciente podem ocorrer (CPPAS, 2018). Um estudo realizado em um hospital universitário de atenção terciária no Município de São Paulo, 2015, evidenciou que para um cuidado seguro, todos os pacientes devem fazer uso da pulseira de identificação com informações corretas e que os profissionais devem realizar a conferência antes de prestar cuidados (Lemos \& Cunha, 2017).

As ferramentas didáticas são materiais utilizados pelo docente para auxiliar o ensino e a aprendizagem de estudantes em relação ao conteúdo proposto. São de fundamental importância no processo de desenvolvimento cognitivo e devem ter o poder de aproximar o aluno do conteúdo, facilitando a efetiva fixação (Silva, et al., 2017).

Define-se mnemônica como o "que ajuda a memória". Presume-se que a técnica mnemônica ajuda a memorizar. Os primeiros documentos que tratam do assunto datam da época do Império Romano perpassando pela Grécia antiga, onde era comum memorizar imensos discursos. Na Idade Média, com os escritos de Tomás de Aquino, chegando ao século XV com os manuais específicos sobre memória bem conhecidos entre os professores do século XXI, por se tratar do alfabeto onde cada letra 
representa uma imagem. A técnica mnemônica foi ignorada e marginalizada no meio acadêmico do século XVII, voltando a ser empregada novamente no estudo e memorização de conteúdo no final do século XX (Mota \& Diniz, 2017).

O Advanced Trauma Life Support (ATLS) é um exemplo de curso de gerenciamento de pacientes gravemente feridos e tem como base o mnemônico $\mathrm{ABCDE}$, que é utilizado nas emergências traumáticas para estabilizar os sinais vitais, e se fundamenta na identificação de sinais/sintomas e no tratamento de problemas que comprometem a vida do indivíduo (Almeida, et al., 2018). O mnemônico SAMPLER, utilizado no atendimento pré-hospitalar às emergências clínicas, também pode ser útil no processo de abordagem para perguntar sobre as condições médicas do paciente (NAEMT, 2018).

Nessa perspectiva, o método mnemônico facilita significativamente o conhecimento sobre o gerenciamento de pacientes, sobre as habilidades clínicas, a organização e as abordagens prioritárias na atenção à saúde. O intuito dessa ferramenta é redefinir as práticas e consolidar o conhecimento profissional, articulando a teoria e a prática, por meio do processo de formação, o que deriva em uma influência mútua com repercussões no processo de ensino-aprendizagem (Almeida, et al., 2018). A utilização da ferramenta parte do princípio de que aquilo que foi memorizado está associado a outra coisa que já se conhece, permitindo a conexões conscientes entre coisas a serem lembradas. O método mnemônico pode vir a contribuir para minimizar os possíveis eventos adversos (Corrêa, 2013).

Diante do exposto, surge o seguinte questionamento: A utilização de um mnemônico para identificação correta do paciente é capaz de auxiliar na prática profissional dos protocolos das metas internacionais de segurança do paciente? O presente estudo tem como objetivo relatar a experiência de acadêmicas de Enfermagem no desenvolvimento de um Mnemônico sobre identificação do paciente para atividade acadêmica curricular da unidade temática Gerenciamento em Serviços de Saúde sobre Ferramentas da Gestão da Segurança do Paciente.

\section{Metodologia}

Trata-se de um estudo descritivo, com abordagem qualitativa, do tipo relato de experiência (RE) em que apresenta a construção de um mnemônico sobre Segurança do Paciente, que visa auxiliar o processo de trabalho dos profissionais de saúde com objetivo de informar e orientar os cuidados com a segurança do paciente cirúrgico submetido à transfusão sanguínea.

A modalidade de RE é uma narrativa científica relevante na contemporaneidade, por tratar-se de uma construção teóricoprática na qual o sujeito-pesquisador discorre sobre uma determinada temática, com o intuito de explanar os saberes adquiridos na experiência em si, valorizando a explicitação descritiva, interpretativa e compreensiva de fenômenos (Daltro \& Faria, 2019).

O método de relato experiência foi selecionado com o intuito de contribuir para melhoria do cuidado em saúde, através da discussão e proposição de ideias, tendo em vista que a experiência das autoras revelam aspectos importantes, que podem ocasionar reflexões e discussões relevantes na área de atuação selecionada. Instigando outros pesquisadores da área a desenvolverem e ampliarem as pesquisas sobre a temática (UFJF, 2016).

Este relato é resultado de atividades do Período Letivo Suplementar (PLS), na modalidade ensino remoto, durante a pandemia da Covid-19 no ano de 2020, produzido por acadêmicas e docentes do Módulo VIII - Gerenciamento em Serviços de Saúde - sobre Ferramentas da Gestão da Segurança do Paciente da Faculdade de Enfermagem Nossa Senhora das Graças (FENSG) da Universidade de Pernambuco.

A unidade temática tem como foco o Gerenciamento em Serviços de Saúde. A forma de avaliação dos alunos no PLS era realizada através da participação nas aulas por meio do Google Meet e do desenvolvimento de atividades síncronas e assíncronas sobre os conteúdos previstos no cronograma. Uma dessas atividades relaciona-se às Ferramentas da Gestão da Segurança do Paciente que tinha como objetivo a construção de uma situação problema sobre um fato associado à Identificação do Paciente, Meta 1 da Segurança do Paciente, e aplicação das ferramentas em gestão para a segurança do paciente. Para esse relato foram consideradas as experiências das alunas e docentes no desenvolvimento do Mnemônico. 
As atividades foram construídas por meio de reuniões remotas, onde o grupo debateu sobre a temática previamente explorada, e estabeleceu a metodologia que seria utilizada. Fundamentado nos estudos realizados acerca da temática, houve a inicialização da criação da ferramenta, obedecendo as seguintes etapas:

1. Levantamento bibliográfico: O método de busca iniciou-se com a pesquisa dos Descritores em Ciências da Saúde (DeCS), definidos como: "Transfusão de Sangue", "Segurança do Paciente" e "Cuidados de Enfermagem". E a definição do operador booleano AND. Utilizando a palavra-chave "Hemotransfusão" a busca dos materiais foi realizada no Portal Regional da BVS, onde encontrou-se 121 arquivos bibliográficos. A seleção dos artigos que compõem a amostra final, relativo a síntese do conhecimento, foi baseada nas recomendações e critérios do Preferred Reporting Items for Systematic Review and Meta-Analyses: The PRISMA Statement (Galvão, et al., 2015). Também foram realizadas pesquisas no Google Scholar, utilizando o termo "Cuidados de Enfermagem na Hemotransfusão", foram encontrados 2 arquivos bibliográficos potencialmente elegíveis. Quanto aos critérios de inclusão, foram aceitos os estudos originais, publicados na íntegra, com acesso gratuito, no período de 2015 a 2020. Foram excluídas as pesquisas publicadas em formato de teses, dissertações, artigos de revisão, relatos de experiências, resumos expandidos e casos clínicos. Segue abaixo, na Figura 1, o processo de escolha dos estudos utilizados como base na criação do mnemônico.

Figura 1 - Fluxograma de seleção dos artigos incluídos na fase do levantamento bibliográfico.

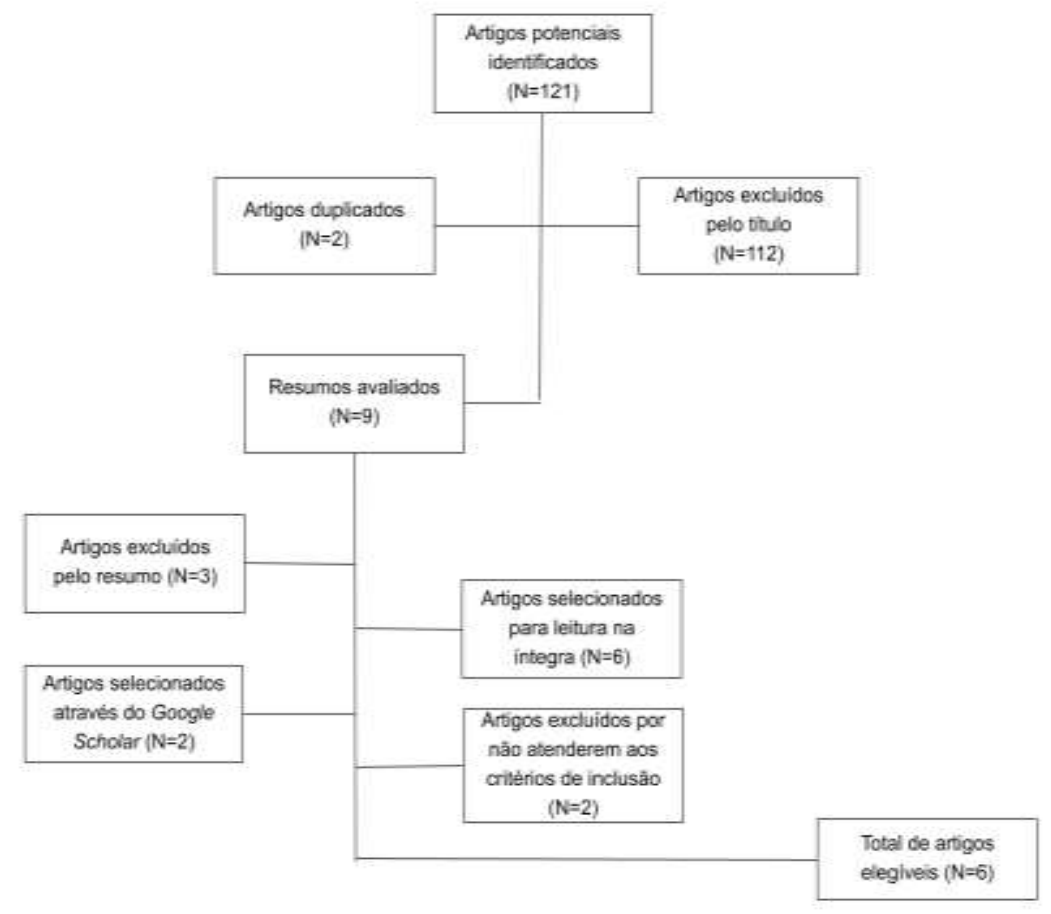

Fonte: Autores (Recife-PE, Brasil - 2021)

\section{Produção do mnemônico:}

2.1 Foram elencados os tópicos fundamentais, em ordem cronológica, para uma boa assistência ao paciente cirúrgico submetido a uma transfusão sanguínea.

2.2 Definiu-se a palavra "ISSO" como regente do mnemônico, a fim de facilitar a memorização do mesmo.

2.3 Posteriormente, foi organizado e associado cada letra da palavra escolhida para a etapa correspondente ao processo de hemotransfusão. 
2.4 Elaborou-se a produção gráfica do mnemônico e o conteúdo didático, previamente selecionado, foi adicionado ao template.

2.5 Com o intuito de fortalecer a segurança na assistência, promover interatividade do profissional e assegurar a realização das etapas, foi adicionado um checklist dividido nas fases perioperatórias.

3. Design do mnemônico: A produção gráfica ocorreu através do Canva, plataforma online de design gráfico, uma ferramenta que permite, de forma simples, a criação de peças gráficas. Como elementos verbais, foram adicionados os conteúdos bibliográficos de forma sucinta, dispostos em página única, no formato apresentação. No círculo cromático foram selecionadas as cores preta, branca e azul, comunicando assim, segurança, profissionalismo e simplicidade. Todos os elementos visuais foram retirados da plataforma.

4. Avaliação: O material foi apresentado aos docentes e demais discentes que compõem a turma, através da plataforma Google Meet. As professoras responsáveis pela disciplina avaliaram o produto de acordo com os critérios previamente estabelecidos, conteúdo bibliográfico, criatividade e aplicabilidade prática. A apresentação ocorreu através da explanação das ferramentas, introdução à temática e explicação das etapas para sua elaboração.

Por tratar-se de um estudo do tipo relato de experiência, descrevendo uma atividade de produção acadêmica, com finalidade exclusiva de educação, ensino ou treinamento, de acordo com o Art. $1^{\circ}$ da Resolução $n^{\circ} 510$ de 07/04/2016, o presente estudo não foi submetido à avaliação pelo sistema CEP/CONEP.

\section{Resultados}

Como processo construtivo da unidade temática de Segurança do Paciente as docentes propuseram aos discentes, do oitavo módulo do curso de Enfermagem, a elaboração de um caso fictício e, posteriormente, uma ferramenta para uso na Gestão em Saúde a fim de minimizar os eventos adversos através de método elementar, direcionado a temática definida pelo grupo de estudantes e baseada na Meta de Identificação do Paciente.

A situação hipotética foi desenvolvida através de uma simulação onde dois pacientes com as mesmas iniciais deram entrada no mesmo hospital necessitando de transfusão sanguínea, ambos foram identificados apenas pelas suas iniciais e possuíam tipo sanguíneo e fator Rh divergentes. Diante da sobrecarga, falta de atenção e comunicação dos profissionais houve a troca das bolsas de sangue e, seguidamente, houve a infusão. $\mathrm{O}$ acontecido foi constatado pelo enfermeiro após a ocorrência do evento adverso no qual desencadeou óbito em um dos pacientes.

Perante a problemática, foi levantada, como fator de promoção à cultura de segurança do paciente, a necessidade da produção de ferramentas acessíveis para auxiliar os profissionais envolvidos na assistência. A técnica do mnemônico foi escolhida pela sua eficácia em facilitar a memorização do conteúdo, complementado pelo checklist que tem o intuito de atestar o cumprimento das etapas conforme o programado.

Para criação do mnemônico e do checklist, foram realizadas pesquisas sobre a temática, analisando os principais tópicos para assegurar a segurança do paciente, a fim de evitar a incorreção por parte da equipe de saúde, o que pode gerar danos irreversíveis ao paciente, como na situação hipotética apresentada anteriormente. Portanto, as informações foram organizadas e adicionadas em ordem cronológica, com o intuito de facilitar o processo de trabalho do profissional. No mnemônico utilizou-se a palavra "ISSO", ao lado de cada letra foi adicionado, em ordem, a conduta que a equipe deverá realizar com o paciente cirúrgico que for submetido à transfusão sanguínea.

Para o checklist dos cuidados de Enfermagem na hemoterapia, optou-se por realizar a divisão em antes, durante e após a infusão do hemocomponente. Compreendendo a importância de cada fase e atentando às necessidades específicas, foram 
identificados os principais procedimentos e descritos de forma sucinta, ao lado de um pequeno quadrado que deverá ser preenchido após checagem da ação descrita.

A entrega da atividade, através Google Meet, foi realizada por meio de uma apresentação elaborada pelas integrantes do grupo para as docentes responsáveis pela unidade temática e para os discentes que compõem a turma. O produto elaborado pode ser visualizado na Figura 2.

Figura 2 - Mnemônico “ISSO”.

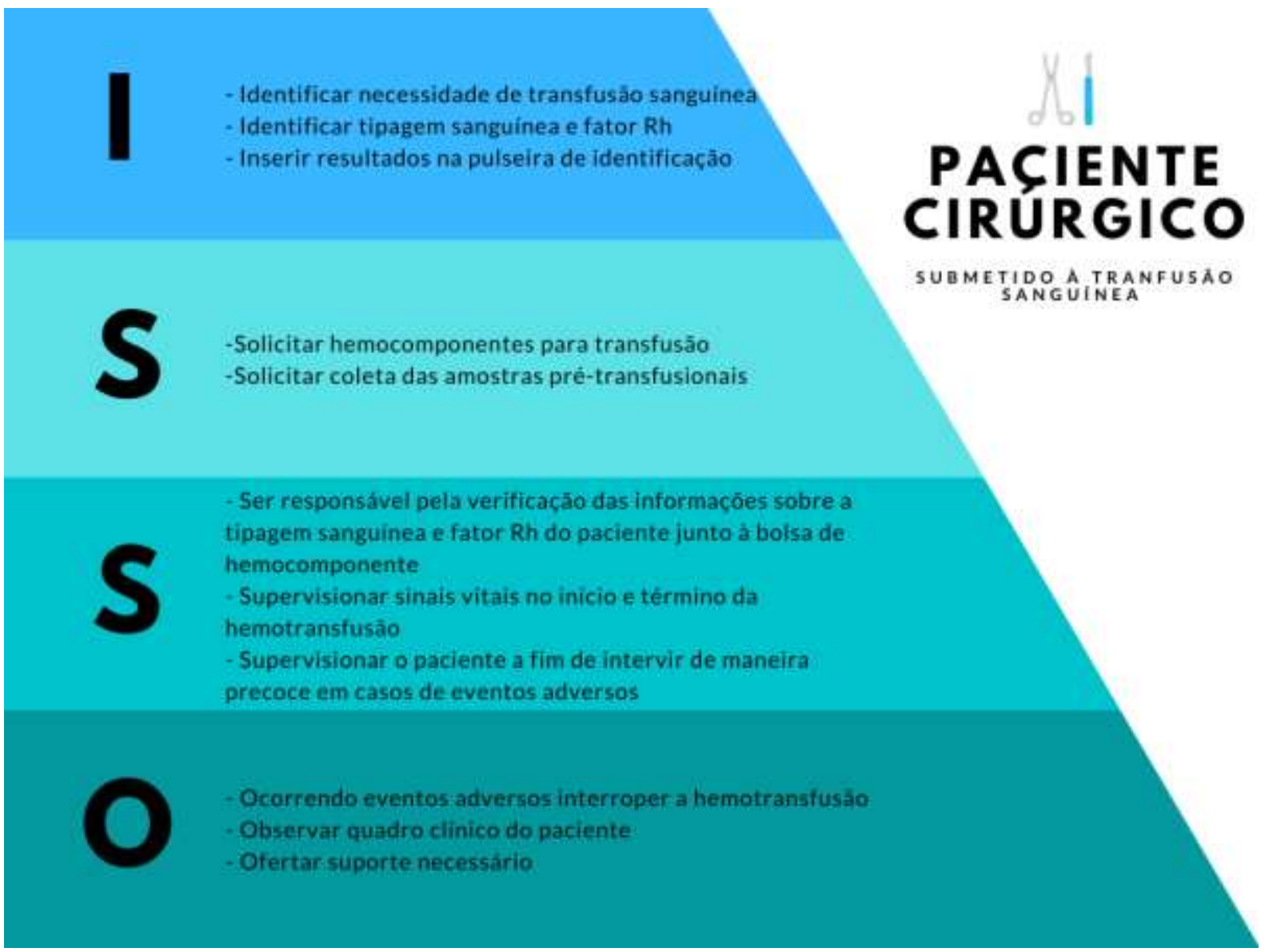

Fonte: Autores (Recife - PE, Brasil, 2021)

\section{Discussão}

Para manter a qualidade na assistência à saúde, é imprescindível que a segurança do paciente esteja pautada como principal estratégia a ser adotada em toda e qualquer assistência de enfermagem. Dito isso, a hemotransfusão é um procedimento que exige vigilância em todas as etapas. Contudo, um estudo realizado em um hospital público de ensino no interior de Minas Gerais, aponta falhas tanto no processo de hemovigilância, quanto na notificação de eventos adversos oriundos de reações transfusionais (Garcia, 2020).

De acordo com a Food and Drug Administration (FDA), responsável por regular como as doações de sangue são coletadas e como o sangue e componentes são transfundidos nos Estados Unidos, em relação às reações hemolíticas transfusionais incompatíveis com ABO, o erro é mais frequentemente causado por erros de identificação evitáveis de amostras de pacientes e identificação do paciente no momento da transfusão (Hensley, et al., 2019).

Neste contexto, destaca-se o Enfermeiro pelo seu cuidado integral ao paciente, atrelado ao conhecimento científico e ao seu papel na segurança transfusional, bem como o Técnico de Enfermagem com as atribuições previstas na Lei do Exercício 
Profissional. O Conselho Federal de Enfermagem (COFEN) normatiza através da Resolução nº 629/2020 a atuação dos Enfermeiros e Técnicos de Enfermagem em Hemoterapia na coleta, armazenamento, controle de qualidade, assistência a doadores e pacientes, além de outras atividades, visando à segurança do paciente (Cofen, 2020).

Através da unidade temática de Gerenciamento em Serviços de Saúde compreendeu-se a importância da criação e adoção das ferramentas para auxiliar as organizações de atenção à saúde, nas ações de gerenciamento, viabilizando um fluxo de trabalho mais seguro e produtivo. Possibilitando a identificação, compreensão e resolução de problemas. Os estudos analisados evidenciaram a importância dessas ferramentas como instrumentos da gestão de qualidade, servindo também como respaldo para a tomada de decisão (Galdino, et al., 2016).

Com isso, a atividade proposta possibilitou a práxis da necessidade de inclusão desses instrumentos nos serviços, colaborando com o processo de trabalho de todos envolvidos na equipe, assegurando a segurança do paciente e objetivando a manutenção da qualidade da assistência, garantindo um cuidado seguro. Porém, sua implementação enfrenta algumas adversidades, dentre elas, podemos citar, a adesão e engajamento dos profissionais que estão na linha de frente das unidades de saúde (Fioreti, et al., 2016).

Dessa forma, no âmbito da transfusão de hemocomponentes e hemoderivados, temática abordada na situação problema formulada e anteriormente citada, foi possível perceber o grande impacto resultante desse processo para o receptor. O processo de hemoterapia é dividido em etapas conhecidas como ciclo do sangue, nas quais a hemovigilância atua veementemente em cada fase, com o intuito de minimizar consideravelmente possíveis danos à saúde. Para obtenção de êxito nesse processo, é fundamental a educação permanente dos profissionais, um ótimo método é o fomento das ferramentas de gestão, como ação estratégica para promoção da segurança do paciente (Frazão, et al., 2019).

É importante dizer que, através da portaria № 529, DE $1^{\circ}$ DE ABRIL DE 2013, a Organização Mundial de Saúde (OMS) definiu segurança do paciente como a redução a um mínimo aceitável, o risco de danos desnecessários associados aos procedimentos de saúde. Em vista disso, mesmo que existam riscos que possam surgir através da assistência à saúde, é importante haver estratégias com o intuito de sanar a possibilidade de que o paciente venha a sofrer com danos desnecessários e evitáveis (Ministério da Saúde, 2014).

Tendo o paciente como sujeito essencial no processo de assistência à saúde, cada vez mais vem surgindo estratégias e ferramentas a fim de garantir segurança nos procedimentos. A Hemovigilância é um processo relativamente recente, definido como um conjunto de estratégias de vigilância presente em todas as etapas do ciclo do sangue, indo desde a captação, seleção e qualificação do doador, até o acompanhamento pós-transfusional dos destinatários. Seu alvo é identificar eventos adversos, prevenir o surgimento, melhorar a qualidade do processo, acarretando maior segurança ao doador e receptor (Anvisa, 2015).

Diante do exposto até aqui, é compreensível a importância das ferramentas de gestão no manejo da segurança do paciente. A partir disso deu-se a ideia de elaborar o Mnemônico presente neste estudo, como uma ferramenta para atuar na educação permanente em saúde (EPS), que nada mais é do que estratégias que aproximam a educação do cotidiano de trabalho, envolvendo a educação problematizadora e as metodologias ativas de aprendizagem (Signor, et al., 2015).

Observa-se ainda, a ascensão da função do enfermeiro no gerenciamento dos serviços em saúde, pelo seu cuidado holístico e integral com o ser humano, sendo primordial o desenvolvimento de competências facilitadoras do processo de trabalho, entre estas, encontram-se a liderança, comunicação e tomada de decisão. Historicamente há uma dicotomia entre a enfermagem assistencial e gerencial, esse fator pode ser observado ainda na graduação, onde a maioria das competências desenvolvidas estão relacionadas ao cuidado direto ao paciente, o que reverbera num desencontro com as exigências do mercado de trabalho. Justificando também, a escassez de estudos que abordem a temática (Ribeiro, et al., 2015; Treviso, et al., 2017).

Diante disso, os desafios enfrentados pelo enfermeiro frente à complexidade do exercício da gestão impulsionam o profissional a refletir e ampliar seus conhecimentos sobre a temática. Um dos desafios enfrentados é a gestão de pessoas, pois, 
geralmente, essa relação é julgada como subordinada. Portanto, para promoção de uma relação harmoniosa e de confiança, é fundamental que sejam estimuladas as relações cooperativas, para uma liderança participativa, onde o indivíduo é conduzido genuinamente a cooperar com os demais, através do seu empoderamento e do desenvolvimento de suas competências (Aragão, et al., 2016).

Desse modo, ficou explícito a importância das ferramentas de gestão em saúde para coadjuvar o enfermeiro gestor a atuar como líder e articulador na promoção de uma assistência integral. Sendo um paradigma na organização da rede de atenção à saúde. Logo, entende-se que a mudança da cultura organizacional revela um processo duradouro, que requer a utilização de diversas ferramentas na promoção de uma gestão efetiva (Soder, et al., 2018; Souza \& Vituri, 2018).

O contato com diferentes formas de criação de novas ferramentas de gestão voltadas ao serviço de saúde, abre um leque de possibilidades para gerir de forma criativa e humanizada uma equipe. Estimulando a criação de diversas outras ferramentas que poderão ser úteis na assistência à saúde progredindo para uma excelência na eficácia e eficiência da assistência de Enfermagem.

\section{Considerações Finais}

Este estudo trouxe como produto a criação de uma ferramenta de gestão, o mnemônico denominado ISSO, para a promoção da segurança do paciente, incluindo o paciente cirúrgico, que demanda uma atenção redobrada devido a gravidade do seu estado geral, com a intenção de auxiliar os profissionais da equipe de saúde minimizando a ocorrência de eventos adversos que possam ocorrer durante o processo transfusional. A categorização da assistência à saúde através da criação do mnemônico, traz benefícios aos envolvidos no processo, tanto para equipe de saúde com a otimização do tempo e menores chances para o erro, como para o paciente que estará ainda mais seguro.

Destaca-se também a importância do protagonismo acadêmico na elaboração e criação de ferramentas acessíveis e funcionais para serem utilizadas nas atividades de enfermagem. Essa construção agregou conhecimento aos discentes e docentes envolvidos, pois foram realizadas diversas pesquisas sobre o tema em questão, onde pode-se notar escassez de estudos relacionados ao assunto abordado.

\section{Referências}

Almeida, A. C., Gonçalves, F. R., Bonfim, C. V do, \& Furtado, B. M. A. S. M. (2018). Assistência De Enfermagem Ao Paciente De Emergência/Urgência. Revista de Enfermagem UFPE on line, 12 (12): 3506-3512. 3506-12. 10.5205/1981-8963-v12i12a237472p3506-3512-2018

Aragão, O. C., Teófilo, J. K. S., Netto, J. J. M., Soares, J. S. A., Goyanna, N. F., Cavalcante, A. E. S. (2016). Competências do enfermeiro na gestão hospitalar. Espaço Para A Saúde - Revista de Saúde Pública do Paraná, Londrina, 17 (2), 66-75. http://espacoparasaude.fpp.edu.br/index.php/espacosaude/article/view/286

COFEN - Conselho Federal de Enfermagem. (2020). RESOLUÇÃO COFEN No 629/2020: Aprova e Atualiza a Norma Técnica que dispõe sobre a Atuação de Enfermeiro e de Técnico de Enfermagem em Hemoterapia. http://www.cofen.gov.br/resolucao-cofen-no-629-2020_77883.html

Corrêa, M. A. P. (2013). As técnicas: Conexão Mnemônica. In: MNEMÔNICA. A Arca Livros, cap. 7

CPPAS - Comissão Permanente de Protocolos de Atenção à Saúde da SES-DF. (2018). Segurança do Paciente: identificação do usuário. https://www.saude.df.gov.br/?s=Seguran\%C3\%A7a+do+Paciente\%3A+identifica\%C3\%A7\%C3\%A3o+do+usu\%C3\%A1rio.

Daltro, M. R., Faria, A. A. de. (2019). Relato de experiência: uma narrativa científica na pós-modernidade. Estudos e Pesquisas em Psicologia, Rio de Janeiro, 19 (1), 223-237. http://dx.doi.org/10.12957/epp.2019.43015

Fioreti, F. C. C. F., Manzo, B. F., Montenegro L. C., Corrêa, A. L., Martins, T. C. R. \& Costa, D. M. (2016). Uso de ferramentas de gestão da qualidade com foco na segurança do paciente neonatal. Rev enferm UFPE on line. 10 (11). 3883-91

Frazão, A. C. A. Y., Rolim, G. K., Pantoja, I. P., Martins, L. F., Silva, M. J. R. B., Oliveira, L. F. de. (2019). Hemovigilância: utilização das ferramentas de gestão para a qualidade no processo transfusional. Revista Eletrônica Acervo Saúde/Electronic Journal Collection Health (REAS/EJCH), 11 (10), p. 642-649. https://acervomais.com.br/index.php/saude/article/view/642 
Galdino, S. V., Reis, E. M. B. dos, Santos, C. B., Soares, F. P., Lima F. S., Caldas, J. G., Piedade, M. A. C. R. \& Oliveira, A. S. (2016). Ferramentas de qualidade na gestão dos serviços de saúde: revisão integrativa de literatura. Rev. Gest. Saúde, 07 (Sulp.1). $1023-57$. https://periodicos.unb.br/index.php/rgs/article/view/3569

Galvão, T. F., Pansani, T. S. A., Harrad, D. (2015). Principais itens para relatar Revisões sistemáticas e Meta-análises: a recomendação prisma. Epidemiologia e Serviços de Saúde, 24 (2), 335-342. https://doi.org/10.5123/S1679-49742015000200017

Garcia, J. Segurança do processo transfusional em pacientes cirúrgicos de um hospital público de ensino do interior de Minas Gerais. (2020). Dissertação (Mestrado em Medicina Tropical e Infectologia) - Programa de Pós-Graduação em Medicina Tropical e Infectologia. http://bdtd.uftm.edu.br/handle/tede/1051

Gomes, A. T. L., Salvador, P. T. C. O., Goulart, C. F., Cecilio, S. G., Bethony, M. F. G. (2020). Innovative Methodologies to Teach Patient Safety in Undergraduate Nursing: Scoping Review. Aquichan, 20 (1), e2018. 10.5294/aqui.2020.20.1.8

Hensley, N. B., Koch C. G., Pronovost P. J., Mershon B. H., Boyd J., Franklin S., Moore D.(2019).Wrong-Patient Blood Transfusion Error: Leveraging Technology to Overcome Human Error in Intraoperative Blood Component Administration. The Joint Commission Journal on Quality and Patient Safety, 45 (3), 190-198. 10.1016/j.jcjq.2018.08.010

IBSP - Instituto Brasileiro para Segurança do Paciente. (2020). PNSP - 7 anos da legislação que instituiu o Programa Nacional de Segurança do Paciente. https://www.segurancadopaciente.com.br/protocolo-diretrizes/pnsp-7-anos-da-legislacao-que-instituiu-o-programa-nacional-de-seguranca-do-paciente/

Lemos, C. L., Cunha, K. C. S.(2017). O Uso Da Identificação De Pacientes Em Uma Unidade Hospitalar. Revista de Enfermagem UFPE on line, 11 (1). 130-9. 10.5205/reuol.9978-88449-6-1101201716

Ministério da Saúde.(2014). Documento de referência para o Programa Nacional de Segurança do Paciente. Fundação Oswaldo Cruz; Agência Nacional de Vigilância Sanitária, Brasília:40p. https://portaldeboaspraticas.iff.fiocruz.br/biblioteca/documento-de-referencia-para-o-programa-nacional-de-seguranca-dopaciente/

Mota, E. R. F., \& Diniz, M. S. K. (2017). O Uso De Técnicas Mnemônicas Como Estratégia De Aprendizagem Para Crianças Do Ensino Fundamental I. Anais dos Cursos de Pós-Graduação Lato Sensu UniEVANGÉLICA. 01 (01), 82-100. http://anais.unievangelica.edu.br/index.php/latosensu/article/view/1775

NAEMT - National Association of Emergency Medical Technicians. (2018). AMLS - Atendimento Pré-Hospitalar às Emergências Clínicas. Artmed.

Ribeiro, A. B. A., Reis, R. P., Bezerra, D. G. (2015). Gestão em Saúde Pública: um enfoque no papel do enfermeiro. Revista Brasileira de Ciências da Saúde. 19 (3), 247-252. 10.4034/RBCS.2015.19.03.12

Santos, L. X., Santana, C. C. A. P., Oliveira, A. S. B. (2021). A hemotransfusão sob a perspectiva do cuidado de enfermagem / Hemotransfusion under the perspective of nursing care / La hemotransfusión bajo la perspectiva del cuidado de enfermería. Revista de Pesquisa Universidade Federal do Estado do Rio de Janeiro 13, 65-71. 10.9789/2175-5361.rpcfo.v13.7458

Silva, A. C. M., Freitag, I. H., Tomaselli, M. V. F., \& Barbosa, C. P. (2017). A importância dos recursos didáticos para o processo ensino-aprendizagem. Arquivos do MUDI, 21 (02), 20-31. https://periodicos.uem.br/ojs/index.php/ArqMudi/article/view/38176/pdf

Silva, E. M., Vieira, C. A., Silva, F. O., Ferreira, E. V. (2017). Desafios da enfermagem diante das reações transfusionais / Nursing challenges in responding to transfusion reactions / Retos de la enfermería ante las reacciones transfusionales. Revista Enfermagem UERJ, 25 (11552). http://dx.doi.org/10.12957/reuerj.2017.11552

Soder, R., Oliveira I. C., Silva, L. A. A. da, Santos, J. L. G., Peiter, C. C., Erdmann, A. L. (2018). Desafios da gestão do cuidado na atenção básica: perspectiva da equipe de enfermagem. Enferm. foco, 9 (3), 76-80.: http://revista.cofen.gov.br/index.php/enfermagem/article/view/1496/465

Souza, J. F. de, \& Vituri, D. W. (2019). Notificações de incidentes relacionados à segurança do paciente como ferramenta de gestão para a mudança da cultura organizacional. Revista Eletrônica Acervo Saúde, (17) e237. https://doi.org/10.25248/reas.e237.2019

Souza, V. (2017). Segurança e intervenção: a assistência de enfermagem em hemotransfusão / Safety and intervention: nursing assistance in blood transfusion /Segurança e intervención: la asistencia de enfermería en Hemotransfusion. 126. https://pesquisa.bvsalud.org/portal/resource/pt/biblio-1026846

Torezan, G., \& Souza, E. N. (2010). Transfusão de hemoderivados: os enfermeiros estão preparados para o cuidado peritransfusional? / Transfusion of blood products: are the nurses prepared to care for peritransfusion? Revista de Enfermagem UFPE on line, 4 (2): 658-665, 20100300

Treviso, P., Peres, S. C., Silva, A. D. da, Santos, A. A. dos. (2017). Competências do enfermeiro na gestão do cuidado. Revista de Administração em Saúde, 17 (69), 8-20. http://dx.doi.org/10.23973/ras.69.59

UFJF - Universidade Federal de Juiz de Fora. (2016). Instrutivo Para Elaboração De Relato De Experiência. Universidade Federal de Juiz de Fora Campus Governador Valadares, Instituto de Ciências da Vida, Departamento de Nutrição. https://www.ufjf.br/nutricaogv/files/2016/03/Orienta\%C3\%A7\%C3\%B5esElabora\%C3\%A7\%C3\%A3o-de-Relato-de-Experi\%C3\%AAncia.pdf

Vermieiro, C. A. F. (2000). Assistência de enfermagem na terapêutica transfusional. Departamento de Enfermagem da Universidade de Federal do Paraná. https://periodicos.uninove.br/saude/article/download/1125/893.

Zampollo, N., Contrin, L. M., Beccaria, L. M., Frutuoso, I. S., Rodrigues, A. M. S., \& Wernek, A. L. (2018). Adesão Ao Protocolo De Identificação Do Paciente E Medicação Segura. Revista de Enfermagem UFPE on line, 12 (10), p. 2667-74. https://doi.org/10.5205/1981-8963-v12i10a234885p1129-1139-2018. 